\title{
Thermal metamorphoses of cosmic dust aggregates: experiments by furnace, electrical gas discharge, and radiative heating
}

\author{
Torsten Poppe ${ }^{1}$, Carsten Güttler ${ }^{2}$, and Tilman Springborn ${ }^{2}$ \\ ${ }^{1}$ Blumenstraße 2, 38162 Weddel in Braunschweig, Germany \\ ${ }^{2}$ Institut für Geophysik und extraterrestrische Physik, Technische Universität Braunschweig, \\ Mendelssohnstraße 3, 38106 Braunschweig, Germany
}

(Received July 30, 2008; Revised October 2, 2008; Accepted October 3, 2008; Online published February 12, 2010)

\begin{abstract}
We experimentally investigated thermal modifications of porous dust aggregates composed of micrometersized grains by furnace, electrical discharge, and laser radiation heating. In the furnace, porous $\mathrm{SiO}_{2}$ aggregates of 95\% porosity at first underwent surface diffusion sintering, which led to progressively increasing necking between adjacent particles. Subsequently, viscous flow dissolved the particulate structure of the still porous sample, and finally melting occurred. Exposing aggregates of various grain types to electrical discharges dispersed most of the sample and left it thermally unprocessed. Nevertheless, some material was thermally processed to sintered aggregates and a tiny fraction to solidified melt spherules with diameters of less than $180 \mu \mathrm{m}$ and most with interior bubbles. In comparison, radiative laser heating turned out to be a much more efficient process to produce melt spherules of chondrule size, and voids were rarer than in discharge heating. Besides providing material data for further applications, our work also allows a direct conclusion to be drawn on chondrule formation. Low energetic efficiency and aggregate destruction exclude chondrule formation from loosely-bound aggregates inside hypothetical nebular lightning channels. However, radiative heating of whatever origin, including possible lightning, remains a candidate process of chondrule formation.
\end{abstract}

Key words: Preplanetary dust aggregates, thermal transformations, sintering, chondrule formation, method: laboratory.

\section{Introduction}

We assume that loosely-bound aggregates of micrometersized cosmic grains underwent heating events in the early solar system. This assumption is supported by several aspects of today's understanding of the early solar system. At first, an important fraction of the dust condensed from the gas phase, a process which required an initially hot solar nebula. In this environment, a dust aggregation started; such processes of aggregation have been investigated in detail in order to describe terrestrial planet formation (e.g., Weidenschilling and Cuzzi, 1993). Moreover, meteoritic chondrule formation indicates that there were heating events in the same period and in the same material reservoir that transformed dust aggregates to melt spherules. Today, frictional heating by shocks in the solar nebula is favored among the numerous hypotheses on chondrule formation (Boss, 1996; Ciesla, 2005). With respect to the following, we add that the hypothesis that heating happened by lightning-like gas discharges is still under serious discussion. Finally, a general hint for heating events of cosmic dust in the early solar system are FU Orionis outbursts (e.g., Bell et al., 2000), which are an increase of luminosity for years or decades which solar-type stars undergo in their period of formation.

Copyright (c) The Society of Geomagnetism and Earth, Planetary and Space Sciences (SGEPSS); The Seismological Society of Japan; The Volcanological Society of Japan; The Geodetic Society of Japan; The Japanese Society for Planetary Sciences; TERRAPUB.

doi: $10.5047 /$ eps.2008.10.001
Consequently, thermal transformations of dust grain aggregates are of interest, and we therefore exposed cosmic dust analogous to furnace (Poppe, 2003), electrical discharge (Güttler et al., 2008), and radiative heating (Springborn et al. in preparation). Here, we give a comparative overview over the manifold outcomes and also discuss aspects of chondrule formation as chondrules must be regarded as an important sink of cosmic dust in the early solar system.

\section{Heating in a Furnace}

By a new sample preparation technique described in Poppe (2003), we produced the most porous layers of micrometer-sized particles known to us (95\% porosity). This was done to acount for the fractal or porous properties of early solar system dust aggregates. The particle layers were made from monodisperse $\mathrm{SiO}_{2}$ spheres of $1.5-\mu \mathrm{m}$ diameter on ceramic substrates (Fig. 1, left). Typically, the particles were arranged in chain-like structures, i.e. most particles had two adjacent particles on roughly opposite sides. The samples were heated under atmospheric pressure and with a variation of heating temperature and heating time. Subsequently, they were analyzed by scanning electron microscopy (SEM).

The thermal transformation started with neck formation between adjacent particles (Fig. 1, center). The SEM images were analyzed by measurements of particle and neck diameter, which showed that the necks had grown and the 


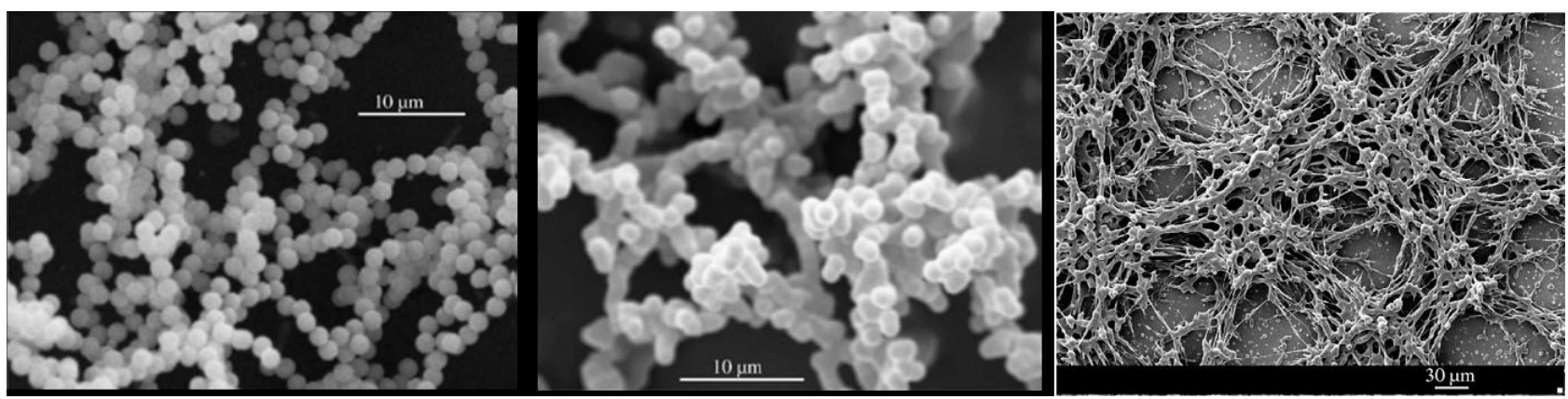

Fig. 1. A thermally unprocessed sample on the left, a sample heated at $1471 \mathrm{~K}$ for $1 \mathrm{~h}$ showing necking between particles (center), and a further processed sample which was transformed by viscous flow and which has lost its particulate structure (right).

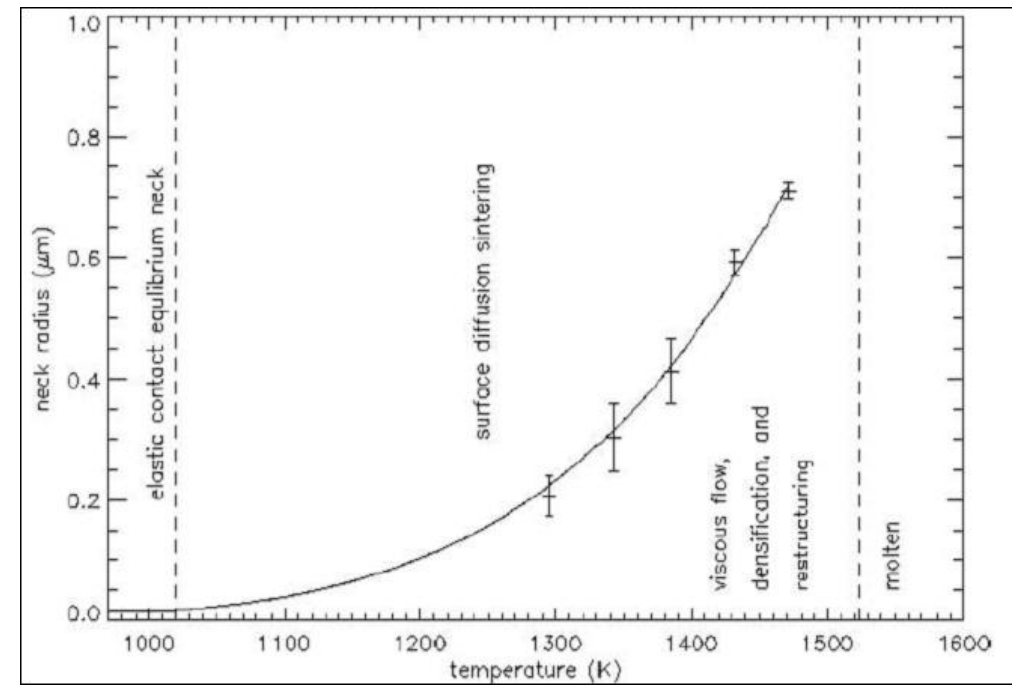

Fig. 2. Temperature-dependent transformations of 1 h-heated samples of $\mathrm{SiO}_{2}$ spheres of $1.5 \mu$ m diameter, measurements of neck diameter as a function of heating temperature, and the fitting function for surface diffusion sintering.

particle diameter determined perpendicular to particle chain direction had become smaller. A surface diffusion sintering model by Nichols and Mullins (1965) was successfully fitted to the neck diameters as a function of heating temperature and heating time. The influence of temperature is by far stronger than the one of time. It was shown (details in Poppe, 2003) that the functional dependence is in agreement with the assumption of surface diffusion sintering, and also the reduced particle radii perpendicular to chain direction indicated the surface as a source of the material transported. Surface diffusion sintering does not change the distance between particle centers, and thus no macroscopic shrinkage should have occurred at this stage of transformation. Figure 2 shows the fit to the heating temperature. With this, constants describing material properties of the $\mathrm{SiO}_{2}$ could be presented in Poppe (2003). Further heating beyond the range of surface diffusion sintering made the particulate structure disappear while the sample still remained porous (Fig. 1, right). This type of transformation could only be explained by viscous flow. Finally, the sample melted, and the melt smoothly covered the ceramic substrate. Figure 2 also summarizes the transformation as a function of temperature for 1-h heated samples. The results allow morphological changes to be predicted according to heating temperature and time for silica. For the more abundant silicates, they are an example for transformations which could occur when heated, but as a consequence of other material constants at other temperature ranges. Such morphological changes are important for the light-scattering properties, mechanical strength, and collisional and aerodynamical properties of early solar system dust aggregates.

\section{Heating in an Electrical Gas Discharge}

The hypothesis of chondrule formation by lightning motivated Wdowiak (1983) to pioneer experimental work on this, and he exposed ground material of the Allende meteorite to a 5-kJ electrical gas discharge. Most of the sample dispersed, and some melt spherules were formed, which solidified as spherules with up to $200 \mu \mathrm{m}$ diameter. The spherules contained bubbles which led Wdowiak (1983) to the conclusion that chondrules did not form by nebular lightning because chondrules were regarded as voidless. The argument of voids against the lightning hypothesis meanwhile appeared weaker than in the past because voids could be caused by low viscosity as a consequence of lower degree of melting than in reality (Maharaj and Hewins, 1993), and voids in chondrules may be rare but are known to exist (Tsuchiyama et al., 2003). Besides this, a progress compared to Wdowiak (1983) seemed possible by performing a series of experiments with varying experi- 


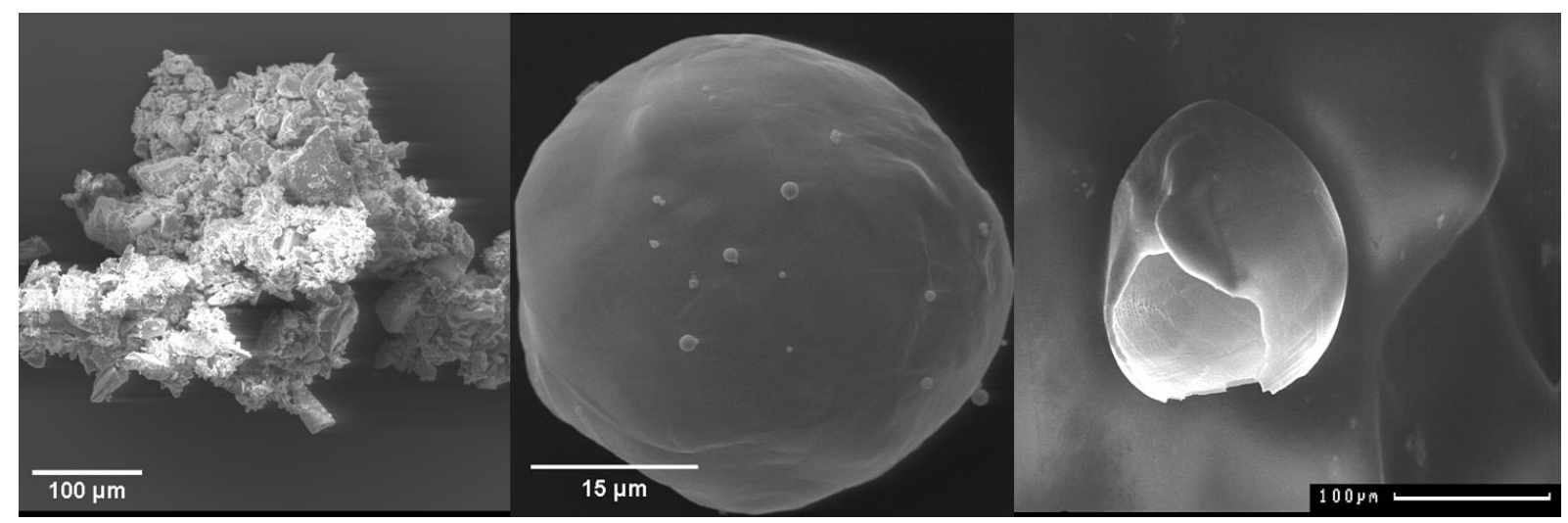

Fig. 3. SEM images of some outcomes of the gas discharge experiments: Aggregate of fayalite grains (left), tiny melt spherule of iron (center), hollow melt spherule of iron (right).

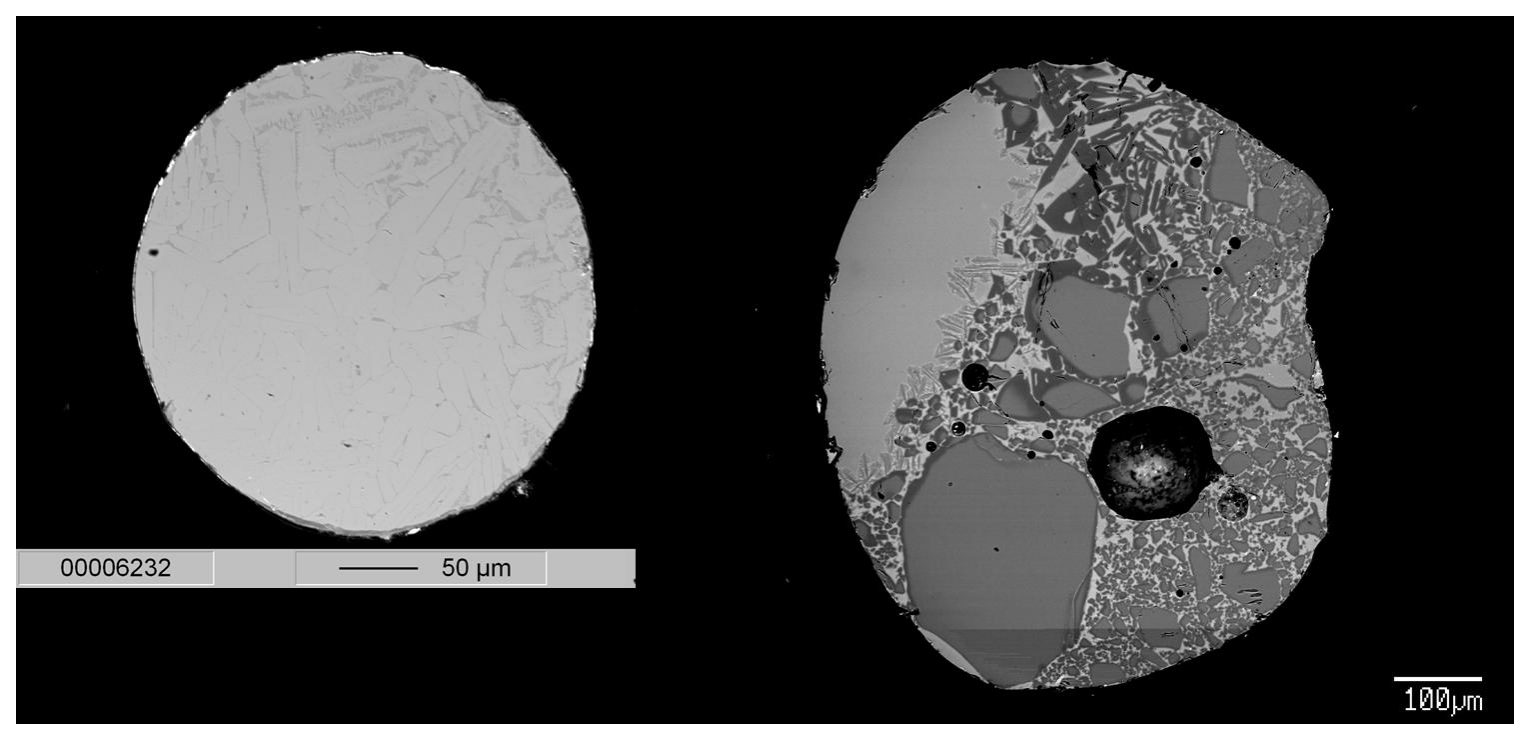

Fig. 4. SEM images of sectioned melt spherules produced by radiative heating. Left: Spherule produced from fayalite grains by $8 \mathrm{~s}$ heating, cooled in free fall. Right: Spherule produced from mantle rock, heated for $25 \mathrm{~s}$, cooled on the ceramic sample holder, which offers a possible explanation for unisotrope texture. Bright areas are pyroxene, darker ones forsterite, the very dark parts are voids.

mental parameters.

We therefore exposed aggregates of various grain types $\left(\mathrm{SiO}_{2}\right.$, olivine, peridotite (earth mantle rock, Witt and Seck, 1987), iron, nickel) to electrical discharges of up to $500 \mathrm{~J}$. The samples were placed between electrodes in a chamber with air pressures between 10 and $10^{5} \mathrm{~Pa}$. In all experimental runs, most of the sample was dispersed and remained thermally unprocessed. Nevertheless, some material was thermally processed to sintered aggregates, and a tiny fraction to solidified melt spherules with diameters less than $180 \mu \mathrm{m}$ and most of them with interior bubbles. Some spherules produced from iron particles were even completely hollow bubbles. Figure 3 shows examples of the manifold phenomenology of experimental outcomes.

The solidified melt spherules were rare, small, and porous. While the porosity, as mentioned above, may be a weak argument against formation in nebular lightnings and one could be tempted to attribute the small size to different length scales in experimental and real situations, an analysis of energetic efficiency shows what "rarity" means in this context. The ratio of energy consumed for melting spherules devided by the discharge energy, yielded values between $0.006 \%$ and $0.06 \%$, depending on the material. Even considering the ultimate source of energy the protoplanetary disk had, which is its potential energy, it is by orders of magnitude too small to explain chondrule formation by nebular lightning. Furthermore, the destruction of aggregates in the gas discharge is a strong argument against chondrule formation inside hypothetical nebular lightning channels. Loosely-bound aggregates would not have survived the even more violent conditions in possible nebular lightning than in the experiments. These are strong and independent arguments against chondrule formation inside nebular lightning channels. However, one must be careful not to misunderstand these arguments. Not only they do not exclude the possibility of nebular lightnings and whatever effect they may have had to dust aggregates, but also they do not exclude that nebular lightning melted chondrule precursors by some mechanism (e.g. radiation or hot gas) outside the discharge channel.

The destruction of loosely-bound aggregates in the electrical gas discharge stresses that, in general, a quiescent en- 
vironment is important for the transformation of dust aggregates to chondrule-like spherules. This reminds us that there is a corresponding challenge to the currently favored hypothesis of chondrule fomation due to frictional heating with gas after passing a shock front. Recently, Sirono (2006) treated this problem.

\section{Radiative Heating by IR Laser}

Powder samples mainly of peridotite (mantle rock) and partially of olivines, including fayalite, obsidian, and albite, were exposed to a $30-\mathrm{W}$ infrared laser beam. Irradiation took from 1 to $30 \mathrm{~s}$, and surrounding air pressures were between $10^{-7} \mathrm{~Pa}$ and ambient atmospheric pressure. The samples were lying on a heat-resistant ceramic holder which could be turned to release the sample into free fall for cooling. In contrast to the gas discharge experiments, we found an efficient transformation. This means that with all types of samples an average of at least $20 \%$ of the sample mass was molten. Concerning the most often used peridotite, on average $70 \%$ of mass was molten, and most of it solidified as spherules of chondrule size (a few $100 \mu \mathrm{m}$ ). This indicates that the energetic efficiency is by order(s) of magnitude better than that found in electrical discharge heating. Only a minor part of the sample was not processed, formed sintered aggregates, or exploded without obvious thermal processing. Furthermore, the spherules had less bubbles, about $10 \%$ void on average, than those produced in the gas discharges. Figure 4 shows two sectioned spherules produced from micrometer-sized powder samples. In Fig. 4 (right), a small void can be seen. The texture indicates mass discrimination of material which was produced from a mixture (mantle rock) in contrast to the spherule left (fayalite).

\section{Summary}

We exposed loosely-bound aggregates of micrometersized dust, which represented properties of early solar system dust, to heating by furnace, electrical discharge, and infrared radiation. This led to manifold outcomes. The comparatively exact setting of heating temperature and time in the furnace allowed the thermal transformation to be resolved as a sequence of neck formation between adjacent particles due to surface diffusion sintering, restructuring, and dissolution of particulate structure by viscous flow, and finally, melting. For several possible applications, the results can be applied to predict morphological changes of dust aggregates according to their heating temperature and time. In contrast to the other experiments, the heating in electrical discharges turned out to be energetically inefficient and violent, dispersing most of the material unprocessed. Only a very few tiny and porous melt spherules or some aggregates with sinter features were found. In con- trast, radiative heating was experimentally shown to efficiently convert dust samples of loosely-bound grains into chondrule-sized melt spherules with far fewer or no voids than those found in discharge heating experiments. Concerning chondrule formation, low energetic efficiency and violent conditions in electrical gas discharges showed that chondrules did not form inside hypothetical lightning channels in the solar nebula, while radiative heating of whatever origin, including lightning, remains a candidate mechanism for chondrule formation. In general, the experiments stress that a quiescent environment is necessary to transform loosely-bound aggregates into chondrules.

Acknowledgments. We thank Dominik Hezel for taking the image in Fig. 4 (left) and John Wasson for support concerning the image in Fig. 4 (right). We also thank the referees Y. Kimura and S. Sirono for their useful comments. Finally, we thank DFG for funding part of the work (grant no Po 817).

\section{References}

Bell, K. R., P. M. Cassen, J. T. Wasson, and D. S. Woolum, The FU Orionis phenomenon and solar nebula material, in Protostars and Planets IV, edited by V. Mannings, A. P. Boss, and S. S. Russell, 897 pp, University of Arizona Press, Tucson, 2000.

Boss, A. P., A concise guide to chondrule formation models, in Chondrules and the Protoplanetary Disk, edited by R. H. Hewins, R. H. Jones, and E. R. D. Scott, 257 pp, Cambridge University Press, Cambridge, 1996.

Ciesla, F. J., Chondrule-forming processes-an overview, in Chondrules and the Protoplanetary Disk, edited by A. N. Krot, E. R. D. Scott, and B. Reipurth, 811 pp, Astronomical Society of the Pacific, San Francisco, 2005

Güttler, C., T. Poppe, J. T. Wasson, and J. Blum, Exposing metal and silicate charges to electrical discharges: Did chondrules form by nebular lightning?, Icarus, 195(1), 504-510, 2008.

Maharaj, S. V. and R. H. Hewins, Vesicles in experimental chondrules as clues to chondrule precursors, Meteoritics, 28(3), 389-390, 1993.

Nichols, F. A. and W. W. Mullins, Morphological changes of a surface of revolution due to capilarity-induced surface diffusion, J. Appl. Phys., 36(6), 1826-1834, 1965.

Poppe, T., Sintering of highly porous silica-particle samples: Analogues of early solar system aggregates, Icarus, 164(1), 139-148, 2003.

Sirono, S., Can a chondrule precursor survive a shock wave?, Astron. Astrophys., 455(1), 379-384, 2006.

Tsuchiyama, A., R. Shigeyoshi, T. Kawabata, T. Nakano, K. Uesugi, and S. Sirono, Three-dimensional structures of chondrules and their highspeed rotation, in Lunar and planetary science XXXIV, pp.1271, 2003.

Wdowiak, T. J., Experimental investigation of electrical discharge formation of chondrules, in Chondrules and their Origin, edited by E. A. King, 279 pp, Lunar and Planetary Institute, Houston, 1983.

Weidenschilling, S. J. and J. N. Cuzzi, Formation of planetesimals in the solar nebula, in Protostars and planets III, edited by E. Levy, J. Lunine, and M. S. Matthews, University of Arizona Press, Tucson, $1031 \mathrm{pp}$, 1993.

Witt, G. and H. A. Seck, Temperature history of sheared mantle xenoliths from the west Eifel, west Germany: Evidence for mantle diapirism beneath the Rhenish Massif, J. Petrol., 28(3), 475-493, 1987.

T. Poppe (e-mail: t.poppe@tu-bs.de), C. Güttler, and T. Springborn 